\title{
Z

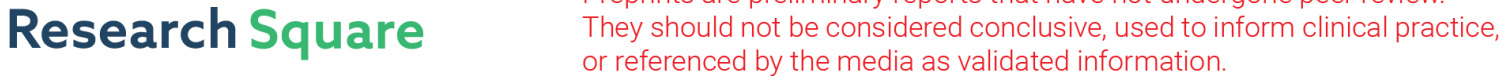 \\ Evolution of Surgical Treatment of Metastatic Spine Tumors.
}

Patricia Zadnik Sullivan ( $\square$ patricia_sullivan@brown.edu )

Brown University Warren Alpert Medical School https://orcid.org/0000-0001-7360-3900

Tianyi Niu

Brown University Warren Alpert Medical School

Jose Fernandez Abinader

Brown University Warren Alpert Medical School

Sohail Syed

Brown University Warren Alpert Medical School

Prakash Sampath

Brown University Warren Alpert Medical School

Albert Telfeian

Brown University Warren Alpert Medical School Jared Fridley

Brown University Warren Alpert Medical School

Petra Klinge

Brown University Warren Alpert Medical School

Joaquin Camara

Brown University Warren Alpert Medical School

\section{Adetokunbo Oyelese}

Brown University Warren Alpert Medical School

\section{Ziya Gokaslan}

Brown University Warren Alpert Medical School

\section{Research Article}

Keywords: metastasis, spine, tumor, cancer, NOMS

Posted Date: January 6th, 2022

DOI: https://doi.org/10.21203/rs.3.rs-1153745/v1

License: (9) (1) This work is licensed under a Creative Commons Attribution 4.0 International License. Read Full License 
Version of Record: A version of this preprint was published at Journal of Neuro-Oncology on March 20th, 2022. See the published version at https://doi.org/10.1007/s11060-022-03982-0. 


\section{Abstract \\ Purpose}

The treatment of cancer has transformed over the past forty years, with medical oncologists, radiation oncologists and surgeons working together to prolong survival times and minimize treatment related morbidity. With each advancement, the risk-benefit scale has been calibrated to provide an accurate assessment of surgical hazard. The goal of this review is to look back at how the role of surgery has evolved with each new medical advance, and to explore the role of surgeons in the future of cancer care.

\section{Methods}

A literature review was conducted, highlighting the key papers guiding surgical management of spinal metastatic lesions.

\section{Conclusion}

The roles of surgery, medical therapy and radiation have evolved over the past forty years, with new advances requiring complex multidisciplinary care.

\section{Defining The Role Of Surgery}

In the 1980s, the role of surgery in the management of spinal metastases was poorly defined. While limited case series supported surgical intervention, these retrospective reports included various pathologies and non-standardized techniques. ${ }^{1-8}$ Oncologic regimens demonstrated variable efficacy, further impacting the measured utility of surgical excision. There was debate in the medical literature regarding the advantage of surgery over radiation alone when reflecting on pooled patient outcomes. ${ }^{9}$ In 1990, the Tokuhashi scoring system was proposed to guide surgical decision making by linking patient pathology and prognosis to treatment. ${ }^{10}$ Even with these advances, given the nature of the studies and selection bias, the role of surgery was not fully assessed.

In 2005, a prospective, randomized clinical trial compared conventional radiation to surgery plus radiation in patients with metastatic spinal disease treated from 1992-2002. ${ }^{11}$ The Patchell trial definitively concluded that surgical decompression and stabilization preserved ambulatory status and prolonged survival. In addition, steroid and opioid pain medication use was reduced in the surgical group, and the study was terminated early due to superiority of surgical treatment. ${ }^{11}$ Further, surgery did not significantly increase hospital length of stay.

While the Patchell study concluded that surgery was superior to radiation alone, this was not the end of the story. The precise type of surgical intervention was still not clear, as the trial inclusion criteria allowed 
the surgeon to tailor his or her approach to meet each patient's specific needs, i.e. laminectomy with or without instrumented fusion. The next step in the evolution of multidisciplinary management was, therefore, to further refine the optimal surgical intervention for patients with metastatic spinal disease.

It was felt that some of the reduced pain medication use among patients in the surgical arm of the Patchell study was likely attributable to stabilization of cancer-related vertebral column instability. The Spine Oncology Study Group (SOSG) proposed the Spinal Instability Neoplastic Score (SINS) following the Patchell study, as a way of identifying patients likely to respond to operative fixation in addition to decompression for metastatic lesions. The SINS system detailed the patient's pain, degree of vertebral body collapse, lytic versus blastic characteristics of the tumor, posterior element involvement, level of disease, and presence of kyphosis or translation related to the level of interest. ${ }^{12}$ Patients were classified as stable, indeterminate or unstable, which guided the use of instrumentation. With this scoring system, spine surgeons were given a framework for how to balance the risk and expense of instrumentation with the benefit of decreased pain and improved stability.

Other surgical considerations not captured by the SINS scoring system or the Patchell study include the vascularity of the tumor and minimally invasive options. The use of preoperative embolization can be considered for highly vascular metastatic lesions to reduce surgical risks. These pathologies include renal cell carcinoma, follicular thyroid cancer, hepatocellular cancer, pheochromocytoma, melanoma, hemangiopericytoma and some sarcomas. Adjuncts such as kyphoplasty have been added to facilitate shorter segment fusions. ${ }^{1-3}$ Laser interstitial thermal therapy (LITT) $)^{16-19}$ and radiofrequency ablation ${ }^{15}$ have also been studied to address metastatic lesions with less morbidity than open cytoreductive approaches.

\section{Advancements In Radiation Therapy}

As surgical decision making has becoming increasingly protocol-driven, the role of radiation therapy has expanded in parallel. Conventional external beam radiation therapy (EBRT), the standard therapy used in the Patchell clinical trial, has been supplanted by newer technologies. Historically, cancer pathologies were divided into very radiation sensitive, moderately sensitive and radioresistant groups, relative to EBRT. Very sensitive lesions included lymphoma, myeloma, and small cell lung cancer (SCLC). Sensitive lesions were breast, prostate, and thyroid pathologies, while radioresistant lesions were colon, renal, melanoma, sarcoma, and non-small cell lung cancers (NSCLC; squamous and adenocarcinoma). The invention of stereotactic radiosurgery (SRS) challenged the sensitive versus resistant classification system, as higher doses of targeted radiation were delivered to index levels of disease in fewer treatments, effectively treating tumors that were previously deemed radioresistant. ${ }^{20,21}$ Using SRS limited the damage to surrounding tissues and decreased the length of the treatment time. ${ }^{21,22}$ Further, SRS provided durable local control rates and improved patient reported quality outcomes post treatment. ${ }^{23-26}$ Protocols were developed to administer EBRT and SRS together for the same patient, with careful monitoring of spinal cord toxicity. ${ }^{27}$ 
Given this expansion of radiation protocols, minimally invasive techniques for surgical decompression gained wider adoption. Prior to SRS, the only effective means to address epidural cord compression was direct surgical excision in patients with radioresistant pathologies. Ventral epidural compression was particularly challenging, as partial and complete corpectomies increased the complexity of the surgical approach. SRS gave spine surgeons a new tool to treat ventral epidural compression for an expanded list of tumor types, and the role or surgery shifted to facilitate a safe radiation treatment field and distance between the tumor and the spinal cord. ${ }^{23-25,28,29}$ This hybrid approach provided durable local control rates for a variety of pathologies. ${ }^{24,28,29}$ An accompanying grading scale, the Bilsky cord compression scale was developed to create a uniform system for assessing the degree of epidural extension of a spinal cord lesion, and to determine eligibility for separation surgery 28,30

With wider use of separation surgery, the limitations of this approach were discovered. Radiation induced myelopathy to the spinal cord can occur when maximal safe doses are exceeded, and patients with recurrence at an index level cannot have unlimited, repeated radiation treatment. ${ }^{31}$ This has complicated multidisciplinary decision making, particularly when a local recurrence occurs. Surgeons and radiation oncologists alike have struggled with when to offer limited surgical resection with separation surgery for projected long-term survivors versus a more aggressive, cytoreductive surgical approach. Furthermore, while studies are limited and of low-quality evidence regarding post-operative wound healing, preoperative radiation is known to compromise skin integrity and disrupt natural tissue planes. ${ }^{32}$ Radiation can also impact rates of postoperative fusion in patients with instrumentation. ${ }^{33}$ Data is limited, however retrospective series suggest that targeted SRS poses lower risk than EBRT. ${ }^{33}$ Radiation can also accelerate the development of vertebral compression fractures (VCF) in patients with lytic lesions or prior EBRT, and the risks may be higher when higher SRS doses are used at a single level. ${ }^{34-36}$ Kyphoplasty may be used as an adjunct to restore vertebral body height in patients with spinal metastases at risk of VCF, further highlighting the necessity of complex, multidisciplinary treatment planning. ${ }^{37-39}$

In an effort synthesize the rapidly evolving treatment recommendations, the NOMS framework was proposed to incorporate the patient's neurologic, oncologic, metastatic and systemic disease status plus radiation considerations into the presurgical evaluation process. ${ }^{40,41}$ The NOMS framework suggests when to offer separation surgery for metastatic lesions, and incorporates the SINS scoring system to consider when instrumented fusion will be required. This scoring system adds complexity to earlier protocols by considering the nuances of radioresistant versus radiosensitive pathologies when EBRT is offered as well as the safety profile of radiation treatment fields.

Prognostically, pathology-specific actuarial scales are widely available to help surgeons determine estimated survival. ${ }^{42-48}$ As patients are surviving longer with metastatic disease, these scoring systems will continue to evolve to reflect treatment options and outcomes data for cancer patients. The NOMS framework has been modified recently to include a molecular typing category, reflecting the impact of targeted therapeutics on prognosis and treatment planning. ${ }^{20}$ Figure 1 provides a case example that 
illustrates these concepts. The patient is a long-term survivor of metastatic RCC and has undergone SRS, separation surgery and fusion over 20 years battling the disease.

\section{Molecular Therapies}

The status of molecular therapeutics for melanoma, NSCLC and RCC is of particular interest for spine surgeons. Patients with these primary tumor types have historically demonstrated poor survival due to poorly controlled systemic disease and radio resistance. While this review will describe the status of targeted therapies for common metastatic pathologies, it is critical to recall that primary and metastatic tumors from the same patient may not share the same genetic mutations. For example, epidermal growth factor receptor (EGFR) mutations seen on biopsy in patients with non-small cell lung cancer may not correlate with an increased response of spinal metastatic lesions to EGFR-tyrosine kinase inhibitors (EGFR-TKI). ${ }^{49}$ Further, some targeted therapies have off-target effects, the most serious of which is treatment-related death (TRD). ${ }^{50}$

\section{Melanoma}

Historically, melanoma metastatic to the spine was associated with a $<4$ month average survival. ${ }^{51}$ This was due to the resistance of metastatic melanoma to chemotherapy, and the ability of this cancer to phenotype switch, acquiring novel genetic mutations to facilitate survival in the face of systemic threats such as hypoxia. In recent years, interleukin-2 as well as drugs targeting PD-1, PD-L1, CTLA-4 and BRAF mutations have demonstrated efficacy in local control rates and overall survival. ${ }^{52-56}$ These drugs have been investigated in small groups of patients with metastatic melanoma in the spine, and have demonstrable efficacy with CNS disease ${ }^{56}$ as well as $40-50 \%$ response rates in patients with metastatic melanoma. ${ }^{55,57}$ For a subset of patients with BRAF mutations within their cancer, BRAF inhibitors such as vemurafenib reduce the risk of death compared with traditional chemotherapy, and improve overall survival. ${ }^{58,59}$ These survival advantages are reported for visceral metastatic melanoma and may not be directly applicable to patients with spinal lesions. Further studies are ongoing comparing combinations of these medications to develop the ideal treatment regimen for patients with metastatic melanoma.

When these therapies are combined with SRS, the response rates in spinal disease are even higher. Specifically, IL-2 therapy coupled with SRS yielded a $62.5 \%$ response rate in a small group of patients with metastatic melanoma in the spine. ${ }^{53}$ In a larger cohort study of patients with metastatic spinal lesions undergoing separation surgery, nine patients with metastatic melanoma demonstrated local recurrence rates under $10 \%$ at follow-up. ${ }^{24}$ Further, a phenomenon known as the abscopal effect may play a role in improved survival and regression of metastatic disease following radiotherapy. ${ }^{60}$ This phenomenon has been seen in patients receiving anti CTLA-4 drugs (ipilimumab) with metastatic melanoma, and may be related to immune-mediated tumor regression at sites distal to the radiation treatment field. ${ }^{60}$

\section{Lung}


Lung cancers are broadly grouped into two categories: small cell lung cancer (SCLC) and non small lung cancer (NSCLC) due to the clinically distinct natures of these two categories. When taken as a monolithic group, the survival for patients with lung cancer metastases to the spine following surgical intervention is quoted in contemporary literature at 2-7.5 months, depending on the type of chemoradiation used to treat the disease. ${ }^{49,61,62}$ However, grouping these pathologies together often complicates the interpretation of outcomes. Modern studies have attempted to delineate both pathological and genotypic subtypes to better understand disease course and prognosis. ${ }^{63}$ More modern studies suggest SCLC with spinal metastases tend to have worse median survival than NSCLC (2 vs $5 \mathrm{mo}) .{ }^{64}$ With respect to treatment modalities, SCLC has historically been considered chemoradiation sensitive, while NSCLC is more resistant to conventional radiation therapy. Even with the limited life expectancy, there is strong data to support surgical intervention to maintain patient quality of life and ambulation. ${ }^{1}$

Targeted therapies are available for NSCLC, with new trials emerging in recent years. Pembrolizumab is a novel PD-1 inhibitor that has demonstrable efficacy against metastatic NSCLC. ${ }^{65}$ EGFR mutations have also been noted within a subset of lung cancers, and EGFR-TKI have also been used to treat patients with metastatic spinal lesions in lung cancer, with mixed results. In a study of 65 patients who underwent surgery for NSCLC metastases to the spine, 35 patients were given the TKI gefitinib or erlotinib in addition to radiation therapy. ${ }^{49}$ Their outcomes were compared to patients receiving traditional chemotherapy, surgery and radiation therapy. No significant differences were seen in survival between these groups, however the authors did report evidence of body regeneration postoperatively in both groups, indicative of successful treatment to the vertebral bodies. ${ }^{49}$

\section{Renal}

Over the past 40 years, drug development and clinical trial and error have led to a sustained improvement in outcomes for patients with metastatic renal cell carcinoma. ${ }^{66,67} 20-60 \%$ of patients with locally advanced renal cell carcinoma (RCC) develop metastases, and patients are grouped into good, intermediate and high risk pools to determine their prognosis and likely response to molecular therapies. ${ }^{68,69}$ Patients with RCC spinal metastases have wide range in reported survival that reflects patient Tokuhashi scores, from 5-32.9 months, according to one recent review. ${ }^{70}$ Currently, nivolumab (PD-1 inhibitor) with or without ipilimumab (CTLA-4 targeted therapy) is the most commonly studied treatment regimen, ${ }^{71-73}$ with vascular endothelial growth factor receptor (VEGFR) TKIs and mammalian target of rapamycin (mTOR) inhibitors for additional targeted therapy. ${ }^{69,74}$ Future studies are needed to determine the optimal combination of drugs and their timing in order to minimize side effects. ${ }^{75,76}$ Targeted therapies like VEGFR-TKIs and mTORis can prolong patient survival, but the prevalence of treatment-related adverse effects is high; up to $98 \%$ in one study. ${ }^{74}$

\section{Conclusion}


Scientific and technological advances have improved the management of spinal metastatic lesions over the past forty years. After the publication of the Patchell trial, surgery was established as a crucial part of metastatic cancer care. In the past ten years, the scope of surgery has been further refined to reflect a patient's symptoms, pathology, and prognosis. Throughout this evolution, surgery has remained the most reliable option for emergent decompression. Despite advances in radiation therapy, SRS with separation surgery requires surgical intervention to be effective in cases of high-grade epidural compression. Molecular targeted therapeutics are emerging for systemic disease, and may supplant invasive surgical procedures, but the optimal combinatorial regimens remain unknown. For this reason, surgeons must remain engaged in medical advancements to guide decision-making and to take the best possible care of patients.

\section{Declarations}

\section{Funding}

The authors declare that no funds, grants, or other support were received during the preparation of this manuscript.

\section{Competing Interests}

Authors PZS, SS, JFA, PK, PS, AT, AO, JF, TN, JC have no relevant financial disclosures.

ZG has the following disclosures: Research Support AO Spine North America, Shareholder Lenoss stock options, Honoraria AO Foundation AOSpine Tumor Knowledge Forum, Board Memberships JNS; Spine Journal: Journal of Spinal Disorders; European Spine Journal; Nature Review; World Neurosurgery; Journal of Spinal Oncology, Advisory Board Proprio

\section{Author Contributions}

All authors contributed to the study conception and design. Material preparation, data collection and analysis were performed by Patricia Zadnik Sullivan and Ziya Gokaslan. The first draft of the manuscript was written by Patricia Zadnik Sullivan and Sohail Syed and all authors commented on previous versions of the manuscript. All authors read and approved the final manuscript.

\section{Ethics Approval}

This is a retrospective literature review. No ethical approval is required.

\section{Consent to publish}

The authors affirm that human research participants provided informed consent for publication of the images at the time of surgical consent.

\section{Data availability}


Data sharing not applicable to this article as no datasets were generated or analysed during the current study.

\section{References}

1. Perrin, R. G. Symptomatic spinal metastases. Am Fam Physician39, 165-172 (1989).

2. Todd, B. Management of painful unstable spinal metastases. Br J Hosp Med43, 328 (1990).

3. Shaw, B., Mansfield, F. L. \& Borges, L. One-stage posterolateral decompression and stabilization for primary and metastatic vertebral tumors in the thoracic and lumbar spine. J Neurosurg70, 405-410 (1989).

4. O'Neil, J., Gardner, V. \& Armstrong, G. Treatment of tumors of the thoracic and lumbar spinal column. Clin Orthop Relat Res227, 103-112 (1988).

5. Kashab, M. \& Böker, D. K. Indication for surgery of spinal metastases within the cervical region. Neurosurg Rev11, 95-97 (1988).

6. Onimus, M., Schraub, S., Bertin, D., Bosset, J. F. \& Guidet, M. Surgical treatment of vertebral metastasis. Spine (Phila Pa 1976)11, 883-891 (1986).

7. Sundaresan, N., Digiacinto, G. V. \& Hughes, J. E. Surgical treatment of spinal metastases. Clin Neurosurg33, 503-522 (1986).

8. Sundaresan, N., Galicich, J. H., Bains, M. S., Martini, N. \& Beattie, E. J. J. Vertebral body resection in the treatment of cancer involving the spine. Cancer53, 1393-1396 (1984).

9. Barcena, A. et al. Spinal metastatic disease: analysis of factors determining functional prognosis and the choice of treatment. Neurosurgery15, 820-827 (1984).

10. Tokuhashi, Y., Matsuzaki, H., Toriyama, S., Kawano, H. \& Ohsaka, S. Scoring system for the preoperative evaluation of metastatic spine tumor prognosis. Spine (Phila Pa 1976)15, 1110-1113 (1990).

11. Patchell, R. A. et al. Direct decompressive surgical resection in the treatment of spinal cord compression caused by metastatic cancer: a randomised trial. Lancet366, 643-648 (2005).

12. Fourney, D. R. et al. Spinal instability neoplastic score: an analysis of reliability and validity from the spine oncology study group. J Clin Onco/29, 3072-3077 (2011).

13. Newman, W. C. et al. Short-segment cement-augmented fixation in open separation surgery of metastatic epidural spinal cord compression: initial experience. Neurosurg Focus50, E11 (2021).

14. Bhatt, A. D., Schuler, J. C., Boakye, M. \& Woo, S. Y. Current and emerging concepts in non-invasive and minimally invasive management of spine metastasis. Cancer Treat ReV39, 142-152 (2013).

15. Zhang, C. et al. Posterior Decompression Surgery and Radiofrequency Ablation Followed by Vertebroplasty in Spinal Metastases from Lung Cancer. Med Sci Monit26, e925169 (2020).

16. Chen, C., Lee, I., Tatsui, C., Elder, T. \& Sloan, A. E. Laser interstitial thermotherapy (LITT) for the treatment of tumors of the brain and spine: a brief review. J Neuroonco/151, 429-442 (2021). 
17. Vega, R. A., Ghia, A. J. \& Tatsui, C. E. Percutaneous Hybrid Therapy for Spinal Metastatic Disease: Laser Interstitial Thermal Therapy and Spinal Stereotactic Radiosurgery. Neurosurg Clin N Am31, 211-219 (2020).

18. Hadzipasic, M., Giantini-Larsen, A. M., Tatsui, C. E. \& Shin, J. H. Emerging Percutaneous Ablative and Radiosurgical Techniques for Treatment of Spinal Metastases. Neurosurg Clin N Am31, 141-150 (2020).

19. Thomas, J. G. et al. A Novel Use of the Intraoperative MRI for Metastatic Spine Tumors: Laser Interstitial Thermal Therapy for Percutaneous Treatment of Epidural Metastatic Spine Disease. Neurosurg Clin N Am28, 513-524 (2017).

20. Caruso, J. P., Cohen-Inbar, O., Bilsky, M. H., Gerszten, P. C. \& Sheehan, J. P. Stereotactic radiosurgery and immunotherapy for metastatic spinal melanoma. Neurosurg Focus38, E6 (2015).

21. Gerszten, P. C., Burton, S. A., Quinn, A. E., Agarwala, S. S. \& Kirkwood, J. M. Radiosurgery for the treatment of spinal melanoma metastases. Stereotact Funct Neurosurg83, 213-221 (2005).

22. Katsoulakis, E., Kumar, K., Laufer, I. \& Yamada, Y. Stereotactic Body Radiotherapy in the Treatment of Spinal Metastases. Semin Radiat Onco/27, 209-217 (2017).

23. Barzilai, O. et al. Predictors of quality of life improvement after surgery for metastatic tumors of the spine: prospective cohort study. Spine J18, 1109-1115 (2018).

24. Laufer, I. et al. Local disease control for spinal metastases following 'separation surgery' and adjuvant hypofractionated or high-dose single-fraction stereotactic radiosurgery: outcome analysis in 186 patients. J Neurosurg Spine18, 207-214 (2013).

25. Lockney, D. T. et al. Spinal stereotactic body radiotherapy following intralesional curettage with separation surgery for initial or salvage chordoma treatment. Neurosurg Focus42, E4 (2017).

26. Redmond, K. J. et al. Consensus guidelines for postoperative stereotactic body radiation therapy for spinal metastases: results of an international survey. J Neurosurg Spine26, 299-306 (2017).

27. Gerszten, P. C., Mendel, E. \& Yamada, Y. Radiotherapy and radiosurgery for metastatic spine disease: what are the options, indications, and outcomes? Spine (Phila Pa 1976)34, S78-92 (2009).

28. Moussazadeh, N., Laufer, I., Yamada, Y. \& Bilsky, M. H. Separation surgery for spinal metastases: effect of spinal radiosurgery on surgical treatment goals. Cancer Contro/21, 168-174 (2014).

29. Barzilai, O. et al. Hybrid Therapy for Metastatic Epidural Spinal Cord Compression: Technique for Separation Surgery and Spine Radiosurgery. Oper Neurosurg (Hagerstown)16, 310-318 (2019).

30. Bilsky, M. \& Smith, M. Surgical approach to epidural spinal cord compression. Hematol Oncol Clin North Am20, 1307-1317 (2006).

31. Sahgal, A. et al. Probabilities of radiation myelopathy specific to stereotactic body radiation therapy to guide safe practice. Int J Radiat Oncol Biol Phys85, 341-347 (2013).

32. Itshayek, E. et al. Timing of surgery and radiotherapy in the management of metastatic spine disease: a systematic review. Int J Onco/36, 533-544 (2010). 
33. Harel, R. et al. Spine instrumentation failure after spine tumor resection and radiation: comparing conventional radiotherapy with stereotactic radiosurgery outcomes. World Neurosurg74, 517-522 (2010).

34. Ling, D. C. et al. Long-Term Outcomes After Stereotactic Radiosurgery for Spine Metastases: Radiation Dose-Response for Late Toxicity. Int J Radiat Oncol Biol Phys101, 602-609 (2018).

35. Jawad, M. S. et al. Vertebral compression fractures after stereotactic body radiation therapy: a large, multi-institutional, multinational evaluation. J Neurosurg Spine24, 928-936 (2016).

36. Roesch, J. et al. Risk for surgical complications after previous stereotactic body radiotherapy of the spine. Radiat Onco/12, 153 (2017).

37. Qian, Z. et al. Kyphoplasty for the treatment of malignant vertebral compression fractures caused by metastases. J Clin Neurosci18, 763-767 (2011).

38. Konig, M. A. et al. Kyphoplasty for lytic tumour lesions of the spine: prospective follow-up of 11 cases from procedure to death. Eur Spine J21, 1873-1879 (2012).

39. Eleraky, M. et al. Balloon kyphoplasty in the treatment of metastatic tumors of the upper thoracic spine. J Neurosurg Spine14, 372-376 (2011).

40. Laufer, I. et al. The NOMS framework: approach to the treatment of spinal metastatic tumors. Oncologist18, 744-751 (2013).

41. Nasrallah, H., Yamada, Y., Laufer, I. \& Bilsky, M. H. A NOMS Framework Solution. Int J Radiat Oncol Biol Phys103, 17-18 (2019).

42. Bollen, L. et al. Predictive Value of Six Prognostic Scoring Systems for Spinal Bone Metastases: An Analysis Based on 1379 Patients. Spine (Phila Pa 1976)41, E155-162 (2016).

43. Cassidy, J. T., Baker, J. F. \& Lenehan, B. The Role of Prognostic Scoring Systems in Assessing Surgical Candidacy for Patients With Vertebral Metastasis: A Narrative Review. Global Spine J8, 638-651 (2018).

44. Choi, D. et al. Prediction Accuracy of Common Prognostic Scoring Systems for Metastatic Spine Disease: Results of a Prospective International Multicentre Study of 1469 Patients. Spine (Phila Pa 1976)43, 1678-1684 (2018).

45. Cui, Y., Lei, M., Pan, Y., Lin, Y. \& Shi, X. Scoring Algorithms for Predicting Survival Prognosis in Patients With Metastatic Spinal Disease: The Current Status and Future Directions. Clin Spine Surg33, 296-306 (2020).

46. Douglas, S., Schild, S. E. \& Rades, D. A new score predicting the survival of patients with spinal cord compression from myeloma. BMC Cancer12, 425 (2012).

47. Rades, D. et al. Prognostic factors in a series of 504 breast cancer patients with metastatic spinal cord compression. Strahlenther Onko/188, 340-345 (2012).

48. Tokuhashi, Y., Uei, H., Oshima, M. \& Ajiro, Y. Scoring system for prediction of metastatic spine tumor prognosis. World J Orthop5, 262-271 (2014). 
49. Lin, H.-H. et al. Functional outcomes and survival after surgical stabilization for inoperable nonsmall-cell lung cancer with spinal metastasis of the thoracic and lumbar spines: a retrospective comparison between epidermal growth factor receptor-tyrosine kinase inhibitor and platinum-based chemotherapy groups. Spinal Cord58, 194-202 (2020).

50. Zhao, J. et al. Treatment-Related Death during Concurrent Chemoradiotherapy for Locally Advanced Non-Small Cell Lung Cancer: A Meta-Analysis of Randomized Studies. PLoS One11, e0157455 (2016).

51. Gokaslan, Z. L., Aladag, M. A. \& Ellerhorst, J. A. Melanoma metastatic to the spine: a review of 133 cases. Melanoma Res10, 78-80 (2000).

52. Brahmer, J. R. et al. Safety and activity of anti-PD-L1 antibody in patients with advanced cancer. $N$ Engl J Med366, 2455-2465 (2012).

53. Seung, S. K. et al. Phase 1 study of stereotactic body radiotherapy and interleukin-2--tumor and immunological responses. Sci Trans/ Med4, 137ra74 (2012).

54. Prieto, P. A. et al. CTLA-4 blockade with ipilimumab: long-term follow-up of 177 patients with metastatic melanoma. Clin Cancer Res18, 2039-2047 (2012).

55. Topalian, S. L. et al. Safety, activity, and immune correlates of anti-PD-1 antibody in cancer. N Engl J Med366, 2443-2454 (2012).

56 . Weber, J. S. et al. Safety and clinical activity of ipilimumab in melanoma patients with brain metastases: retrospective analysis of data from a phase 2 trial. Melanoma Res21, 530-534 (2011).

57. Hamid, O. et al. Safety and tumor responses with lambrolizumab (anti-PD-1) in melanoma. N Engl J Med369, 134-144 (2013).

58. Chapman, P. B. et al. Improved survival with vemurafenib in melanoma with BRAF V600E mutation. $N$ Engl J Med364, 2507-2516 (2011).

59. Flaherty, K. T. et al. Inhibition of mutated, activated BRAF in metastatic melanoma. N Engl J Med363, 809-819 (2010).

60. Postow, M. A. et al. Immunologic correlates of the abscopal effect in a patient with melanoma. $N$ Engl J Med366, 925-931 (2012).

61. Zairi, F. et al. Evaluation of the relevance of surgery in a retrospective case series of patients who underwent the surgical treatment of a symptomatic spine metastasis from lung cancer. Eur Spine J25, 4052-4059 (2016).

62. Truong, V. T. et al. Surgical Intervention for Patients With Spinal Metastasis From Lung Cancer: A Retrospective Study of 87 Cases. Clin Spine Surg34, E133-E140 (2021).

63. Amelot, A. et al. Spinal metastases from lung cancer: Survival depends only on genotype, neurological and personal status, scarcely of surgical resection. Surg Onco/34, 51-56 (2020).

64. Kumar, N. et al. The Influence of Histologic Subtype in Predicting Survival of Lung Cancer Patients With Spinal Metastases. Clin Spine Surg31, E1-E7 (2018). 
65. Gandhi, L. et al. Pembrolizumab plus Chemotherapy in Metastatic Non-Small-Cell Lung Cancer. $N$ Engl J Med378, 2078-2092 (2018).

66. Ishihara, H. et al. Assessing improvements in metastatic renal cell carcinoma systemic treatments from the pre-cytokine to the immune checkpoint inhibitor eras: a retrospective analysis of real-world data. Jpn J Clin Onco/51, 793-801 (2021).

67. Ishihara, H. et al. Prognostic impact of systemic therapy change in metastatic renal cell carcinoma treated with cytoreductive nephrectomy. Jpn J Clin Onco/51, 296-304 (2021).

68. Oza, B. et al. RAMPART: A phase III multi-arm multi-stage trial of adjuvant checkpoint inhibitors in patients with resected primary renal cell carcinoma (RCC) at high or intermediate risk of relapse. Contemp Clin Trials108, 106482 (2021).

69. Salgia, N. J., Dara, Y., Bergerot, P., Salgia, M. \& Pal, S. K. The Changing Landscape of Management of Metastatic Renal Cell Carcinoma: Current Treatment Options and Future Directions. Curr Treat Options Oncol20, 41 (2019).

70. Goodwin, C. R. et al. The Challenges of Renal Cell Carcinoma Metastatic to the Spine: A Systematic Review of Survival and Treatment. Global Spine J8, 517-526 (2018).

71. Alhalabi, O. et al. Outcomes of patients with intermediate-risk or poor-risk metastatic renal cell carcinoma who received their first cycle of nivolumab and ipilimumab in the hospital because of symptomatic disease: The MD Anderson Cancer Center experience. Int J Cancer149, 387-393 (2021).

72. Bando, Y. et al. Treatment outcomes of molecular targeted therapy following nivolumab in metastatic renal cell carcinoma. Jpn J Clin Onco/51, 1313-1318 (2021).

73. Okuno, Y., Tanaka, R., Mikami, K. \& Takeuchi, T. [Renal Cancer Tissue after Nivolumab/Ipilimumab Combination Therapy for Metastatic Renal Cell Carcinoma]. Hinyokika Kiyo66, 13-17 (2020).

74. Tomita, Y. et al. Efficacy and safety of subsequent molecular targeted therapy after immunocheckpoint therapy, retrospective study of Japanese patients with metastatic renal cell carcinoma (AFTER I-O study). Jpn J Clin Onco/51, 966-975 (2021).

75. Aggen, D. H., Drake, C. G. \& Rini, B. I. Targeting PD-1 or PD-L1 in Metastatic Kidney Cancer: Combination Therapy in the First-Line Setting. Clin Cancer Res26, 2087-2095 (2020).

76. Osawa, T. et al. Overview of current and future systemic therapy for metastatic renal cell carcinoma. Jpn J Clin Oncol49, 395-403 (2019).

\section{Figures}



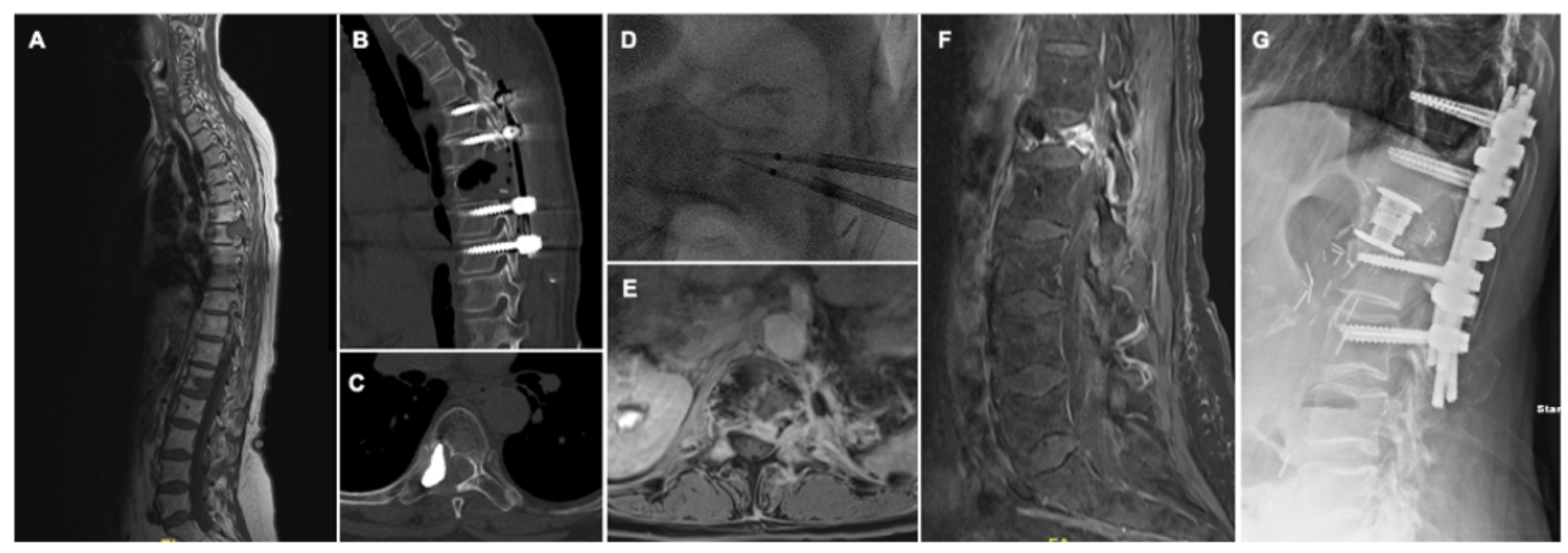

\section{Figure 1}

The patient is a 20-year survivor of metastatic renal clear cell carcinoma (ccRCC). She first underwent a left nephrectomy in 2001, followed by a right sided recurrence of ccRCC and resection of 4 lesions within the kidney ten years later. She subsequently developed spinal metastases in 2013 (A) and underwent Cyberknife SRS to T7 and L1. She developed progressive disease at T7 and underwent embolization, T7 laminectomy and transpedicular partial corpectomy with T5-9 fusion (B-C). This separation surgery facilitated subsequent repeat SRS to the tumor bed. In 2019, she demonstrated progressive disease at L1, and underwent radiofrequency ablation (D) with methymethacrylate augmentation. She developed a worsening compression deformity at L1 and back pain (F) and an L1 corpectomy with cage placement and T11-L3 fusion (G). At two years post corpectomy, her hardware remains intact and there is no evidence of recurrent disease. Throughout her treatment course, she has trialed PD-1 inhibitors as well as TKls with variable responses. 